\section{Liquid biopsy for the identification of intravascular large B-cell lymphoma}

Intravascular large B-cell lymphoma (IVLBCL) is a rare subtype of diffuse large B-cell lymphoma (DLBCL) that lacks lymphadenopathy and extranodal tumors. ${ }^{1}$ For diagnosis, bone marrow biopsies (BMB) and random skin biopsies (RSB) are usually performed. However, due to the small tumor cell content, false negative findings are not uncommon. In recent reports, cell-free DNA (cfDNA) could be helpful for identifying tumor-specific mutations in a variety of neoplasms including malignant lymphomas. ${ }^{2}$ Given the difficulties of diagnosis via conventional biopsies, IVLBCL may be a good candidate to which cfDNA analysis is applied to assist diagnosis. The majority of IVLBCL cases are reportedly non-germinal center B-cell-like (non-GCB). ${ }^{1}$ Thus, mutations are likely to be frequent in genes involved in the B-cell receptor (BCR)-signaling pathway, the Toll-like receptor/Interleukin-1 receptor (TLR/IL-1R) pathway, and the nuclear factor kappa B (NFKB) pathway. ${ }^{2 \cdot 6}$ Here, we report that a mutational analysis of genes via liquid biopsy resulted in high diagnostic yields and effective monitoring of IVLBCL.

This study included 27 IVLBCL patients with archived samples, who were diagnosed with IVLBCL from January 2005 to May 2017 in the participating institutions. Diagnosis of IVLBCL was made at the pathology department of each institution. The study was approved by the institutional review boards of each institution, and conducted according to the Declaration of Helsinki. Tissuederived DNAs (tdDNAs) were available in 26 out of 27 patients (Online Supplementary Figure S1). Paired cfDNAs were also available in 9 out of the 26 patients with tdDNAs. Reference genomic DNAs (gDNAs) were available in 8 out of the 9 patients. In 1 patient, only cfDNA was available.

Targeted sequencing for 8 genes (B2M, BTG2, CARD11, CD79B, MYD88, PIM1, PRDM1, and TNFAIP3) was done in 9 IVLBCL patients with paired samples based on literature data (Online Supplementary Table S1) using the Ion Torrent Personal Genome Machine (Thermo Fisher Scientific). Mutational analysis to detect MYD88 mutation c.794T>C, p.Leu265Pro, (L265P MYD88) was performed using droplet digital PCR (ddPCR) assays (Bio-Rad) for 46 samples collected from 27 patients. See Online Supplementary Methods for further information.

Patient characteristics are summarized in Table 1 and described in more detail in Online Supplementary Table S2. All cases were categorized as the non-GCB according to Hans classification (Online Supplementary Table S3). The diagnostic yield of initial RSB was 78.9\% (16/21). For 1 patient with a negative result at the initial biopsy, a second RSB was performed, which led to the diagnosis of IVLBCL. Initial BMB detected infiltration of lymphoma cells in $48.1 \%(13 / 27)$ patients, but repetition of BMB increased successful detection rate up to $66.7 \%(18 / 27)$. Sensitivity of conventional diagnostic tests in our cohort largely followed the results of a prior report except for a lower detection rate in bone marrow aspiration. ${ }^{7}$ FDG PET/CT $\left({ }^{18} \mathrm{~F}\right.$-fluorodesoxyglucose positron emission tomography/computed tomography) demonstrated increased FDG uptake in $78.6 \%(11 / 14)$ patients.

We extracted cfDNAs from 10 plasma and 28 serum samples at various time points (Online Supplementary Table S4). The median concentrations were $91.3 \mathrm{ng} / \mathrm{ml}$ (range, 19.1-2900.0 ng/ml). Nine cfDNAs from 4 plasma
Table 1. Patient characteristics

\begin{tabular}{|c|c|}
\hline & Parameter value \\
\hline No. of patients & 27 \\
\hline Median age, years (range) & $71(28-88)$ \\
\hline Male sex, n (\%) & $10(37.0)$ \\
\hline \multicolumn{2}{|l|}{ Symptom, n (\%) } \\
\hline Fever & $23(85.2)$ \\
\hline Neurological & $11(40.7)$ \\
\hline Respiratory & $4(14.8)$ \\
\hline Gastro-intestinal & $3(11.1)$ \\
\hline \multicolumn{2}{|l|}{ Clinical course, n (\%) } \\
\hline Primary progressive & $24(88.9)$ \\
\hline Relapsing-remitting episode prior to diagnosis & $3(11.1)$ \\
\hline \multicolumn{2}{|l|}{ Tissue biopsy/aspiration, proven/performed (\%) } \\
\hline Random skin biopsy & $17 / 21(81.0)^{\dagger}$ \\
\hline Bone marrow biopsy & $18 / 27(66.7)^{\ddagger}$ \\
\hline Other biopsies & $10 / 10(100.0) *$ \\
\hline Bone marrow aspiration & $10 / 25(40.0)$ \\
\hline \multicolumn{2}{|l|}{ FDG PET/CT } \\
\hline Positive, n (\%) & $11(78.6)$ \\
\hline Negative, n (\%) & $3(21.4)$ \\
\hline Hemophagocytic Lymphohistiocytosis, n (\%) & $12(44.4)$ \\
\hline non-GCB by Hans classification, $\mathrm{n}(\%)$ & $21(100.0)$ \\
\hline \multicolumn{2}{|l|}{ Abnormalities in blood cell counts, $\mathrm{n}(\%)$} \\
\hline Leukocytopenia ${ }^{8}$ & $7(25.9)$ \\
\hline Anemia & $18(66.7)$ \\
\hline Thrombocytopenia & $12(44.4)$ \\
\hline \multicolumn{2}{|l|}{ Laboratory data, median (range) } \\
\hline Lactate dehydrogenase [U/1] & $699(223-5199)$ \\
\hline Soluble interleukin 2 receptor [U/ml] & $5090(595-25012)$ \\
\hline C-reactive protein [mg/dl] & $7.7(0.03-23)$ \\
\hline Albumin [g/dl] & $2.4(1.3-3.7)$ \\
\hline
\end{tabular}

${ }^{1} 16 / 21(76.2 \%)$ at first skin biopsy. ${ }^{\ddagger} 13 / 27$ (48.1\%) at first bone marrow biopsy. ${ }^{*} 13$ sites positive out of 16 sites. ${ }^{.} \mathrm{WBC}$ count $<4000 / \mu \mathrm{L} .{ }^{\mathrm{g}} \mathrm{Hemoglobin}<11 \mathrm{~g} / \mathrm{dl}$. " platelet count $<10 \times 10^{4} / \mu \mathrm{L}$. FDG PET/CT, ${ }^{18} \mathrm{~F}$-fluorodeoxyglucose-positron emission tomography/computed tomography; GCB: germinal center B-cell-like.

and 5 serum samples obtained at diagnosis were analyzed in targeted sequencing.

Targeted sequencing for 8 genes in 9 patients demonstrated at least 1 mutation in CD79B, MYD88, PIM1, $P R D M 1$, or BTG2 in both tdDNAs and cfDNAs, or only in cfDNAs (Figure 1A, 1B, and Online Supplementary Table S5). Sequencing coverage of $\geq \times 100$ in cfDNAs, that of $\geq$ $\times 500$ in tdDNAs, and that of $\geq \times 100$ in reference gDNAs were $78.3-88.8 \%, 82.9-90.6 \%$, and $83.1-91.5 \%$, respectively (Online Supplementary Table S6). We identified 3 missense $C D 79 B$ mutations (p.Tyr196Cys, p.Tyr196His, and p.Leu199Pro) in 6 out of $9(66.7 \%)$ patients. All MYD 88 mutations, which were found in 5 of $9(55.6 \%)$ patients, were L265P. Sixteen missense PIM1 mutations, 2 nonsense/ 2 missense PRDM1 mutations, and 2 missense BTG2 mutations were each seen in $6(66.7 \%), 4$ $(44.4 \%)$, and $2(22.2 \%)$ patients, respectively. Mutation enrichment in WRCY motifs and/or C:G base pair implied that the PIM1 mutations were associated with aberrant somatic hypermutations (aSHM) (Online Supplementary Table S7). Importantly, variant allele fre- 


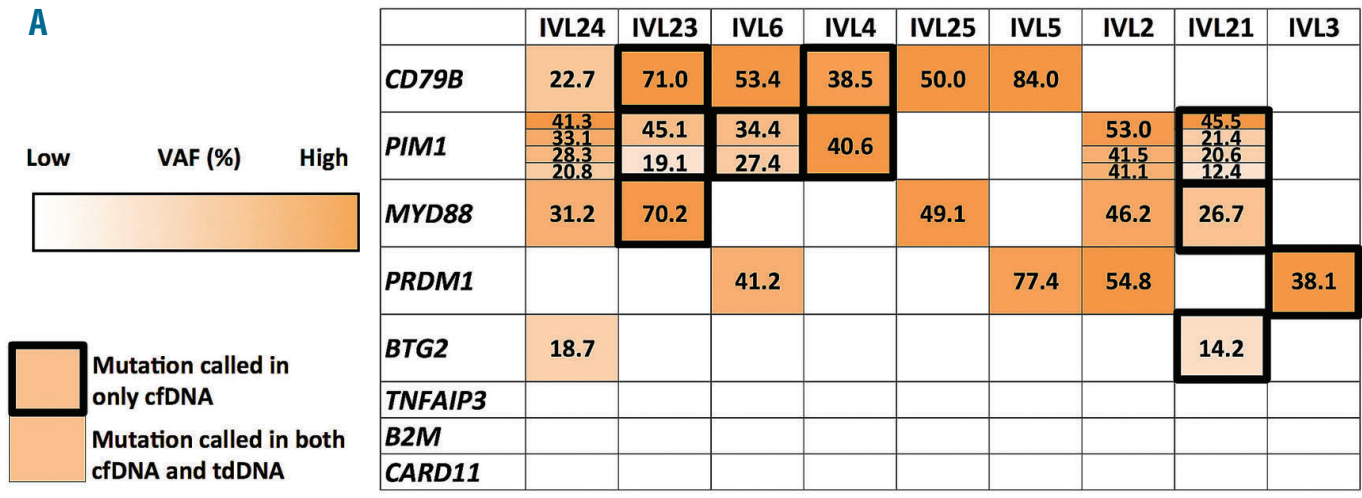

B

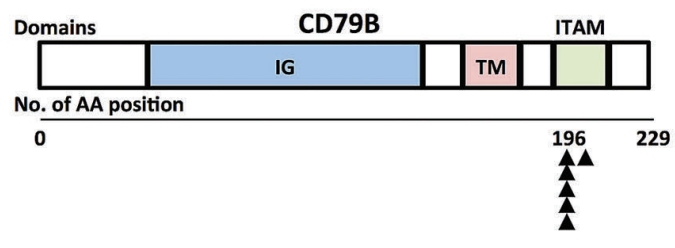

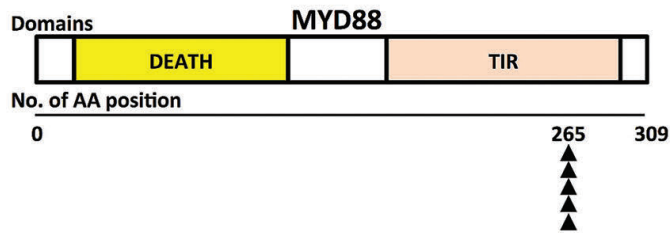

Immunoglobulin

Transmembrane domain

Immunoreceptor tyrosine-based activation motif

Death domain

Toll-interleukin1-resistance $\square$ Nonsense mutation

Serine/Threonine protein kinases, catalytic domain

SET (Su(var)3-9, Enhancer-of-zeste, Trithorax) domain

Zinc finger $\mathrm{C2}$ 2-type

Domains

PIM1
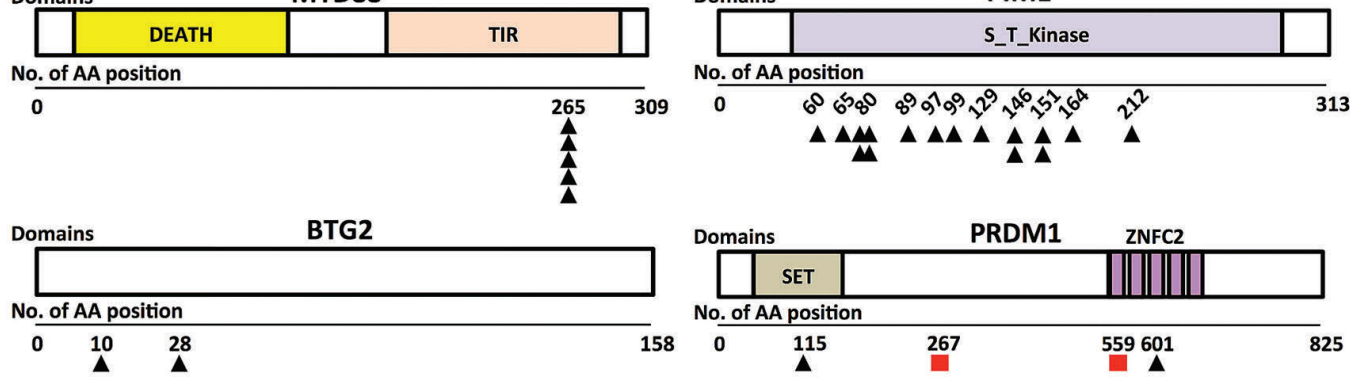

C

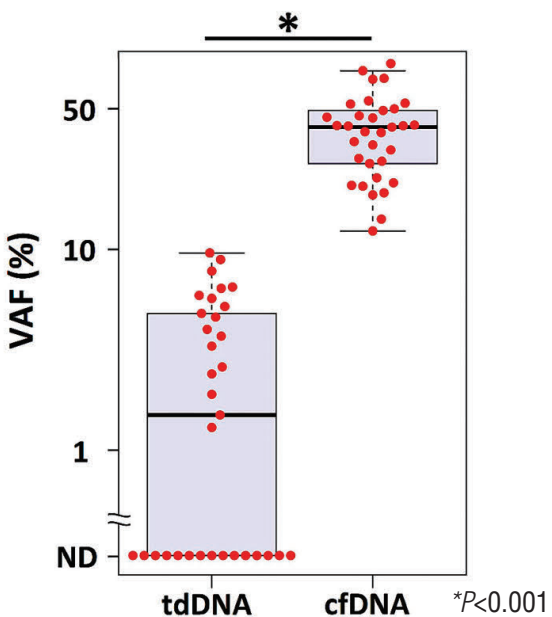

D

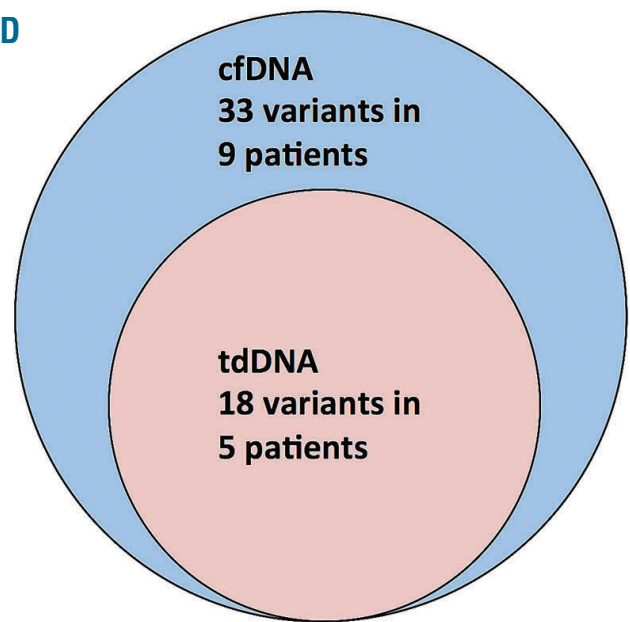

Figure 1. Results of targeted sequencing in 9 patients using cell-free DNAs, tissue-derived DNAs, and reference genomic DNAs.A. Summary of gene alterations in 9 IVLBCL patients. Numbers in boxes represent variant allele frequencies (\%) in cell-free DNAs (cfDNAs). Mutations found in only cfDNAs are surrounded by black thick outline. Variants are color-coded according to their variant allele frequencies of cfDNAs. VAF: Variant allele frequencies; cfDNA: cell-free DNA; tdDNA: tissue-derived DNA. B. The positions of mutations in each gene at protein levels. A black triangle indicates missense mutation. A red box indicates nonsense mutation. IG, Immunoglobulin; TM, Transmembrane domain; ITAM, Immunoreceptor tyrosine-based activation motif; DEATH, Death domain; TIR, Toll-interleukin1-resistance; S_T_Kinase, Serine/Threonine protein kinases, catalytic domain; SET, Su(var)3-9, Enhancer-of-zeste, Trithorax domain; ZNFC2, Zinc-finger $\mathrm{C} 2 \mathrm{H} 2$-type; AA, Amino acid. C. Comparison of variant allele frequencies in tissue-derived DNAs with those in cell-free DNAs. VAF, Variant allele frequencies; tdDNA, tissue derived DNA; cfDNA, cell-free DNA. D. Venn diagram of variants called in tissue-derived DNAs with those in cell-free DNAs; tdDNA: tissue-derived DNA; cfDNA: cell-free DNA. 
quencies (VAFs) were significantly higher in cfDNAs than in tdDNAs (mean, $39.8 \%$ vs. $2.6 \% ; P<0.001$ ) (Figure 1C). In 5 patients, 15 variants were called in cfDNAs, but not in tdDNAs (Figure 1D). On the other hand, all variants detected in tdDNAs were confirmed in cfDNAs.

The ddPCR assay for L265P MYD88 was performed with all 46 samples including tdDNAs and cfDNAs. This revealed L265P MYD88 in 5/10 (50.0\%) cfDNA samples and $19 / 36$ (52.8\%) tdDNA samples from 16/27 (59.3\%) patients with IVLBCL (Online Supplementary Table S8). The mean VAF of L265P MYD88 in the mutation-positive samples was $49.3 \%(26.7-81.3 \%)$ in cfDNAs and $6.5 \%$ $(0.21 \%-34.8 \%)$ in tdDNAs (Online Supplementary Figure S2).

Longitudinal analysis of cfDNAs was performed in 4 patients (Figure 2). L265P MYD88 was detectable in cfDNA from one patient (IVL2) 117 days before the definite diagnosis of IVLBCL, when $B M B$ resulted in a negative pathological finding (Figure 2A). Symptoms in this patient disappeared spontaneously without any therapy after initial presentation. The mutation was also detected in cfDNAs collected during the afebrile period, although VAFs were much lower. Mutations were detectable in cfDNAs prior to definite diagnosis of relapse in 3 patients (Figure 2B-2D).

Comparative analysis of $6 \mathrm{cfDNA}$ pairs extracted from serum and plasma samples (Online Supplementary Table S4) demonstrated higher concentrations and lower VAFs in serum (Online Supplementary Figure S3-S4).

Here, we described how gene mutations affecting the BCR/TLR/IL-1R/NFKB signaling pathways were frequent in patients with IVLBCL. L265P MYD88 was particularly frequent. This mutation has been detected in most cases of Waldenström macroglobulinemia ${ }^{1}$ and $22-32.5 \%$ of non-GCB type DLBCL. ${ }^{4,8,9}$ A higher rate of L265P MYD 88 has been reported in some subtypes of extranodal DLBCL: primary central nervous system lymphoma (PCNSL, more than $50 \%$ ), primary cutaneous DLBCL,
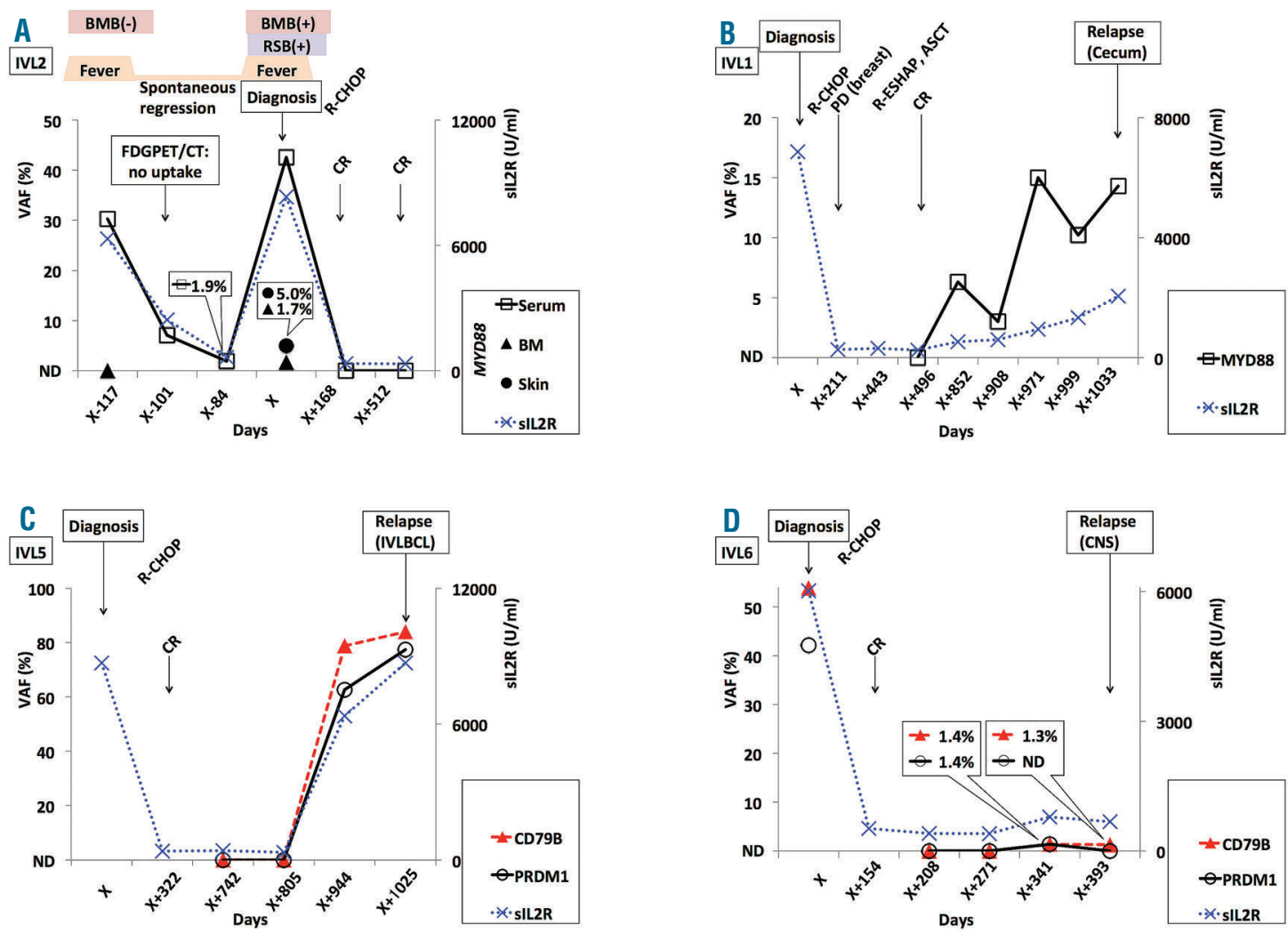

Figure 2. Longitudinal analysis of cell-free DNA. A. L265P MYD88 mutation was detectable prior to a definite diagnosis of intravascular large B-cell lymphoma (IVL2). White squares with solid black line represent MYD88 in cell-free DNAs; Black triangles represent MYD88 in tissue-derived DNAs prepared from bone marrow cells; A black circle represents MYD88 in tissue-derived DNAs prepared from a skin FFPE sample; Blue X-marks with dotted line represent levels of soluble interleukin-2 receptor. VAF, Variant allele frequencies; BM, Bone marrow; sIL2R, soluble interleukin-2 receptor; BMB, bone marrow biopsy; RSB, random skin biopsy; FDG PET/CT, ${ }^{18} \mathrm{~F}$-fluorodesoxyglucose positron emission tomography/ computed tomography; R-CHOP, rituximab, cyclophosphamide, doxorubicin, vincristine, prednisone; CR: complete response; ND, Not detected. B. L265P MYD88 mutation was detectable prior to relapse as extranodal diffuse large B-cell lymphoma of the cecum in a patient (IVL1). White squares with solid black line represent L265P MYD88 mutation in cell-free DNAs; Blue X-marks with dotted line represent levels of soluble interleukin-2 receptor. VAF, Variant allele frequencies; sIL2R, soluble interleukin-2 receptor; R-CHOP, rituximab, cyclophosphamide, doxorubicin, vincristine, prednisone; PD, progressive disease; R-ESHAP, rituximab, etoposide, cytarabine, cisplatinum, and methylprednisolone; ASCT, autologous stem cell transplantation; CR, complete response; ND: not detected. C. Y196C CD79B and PRDM1 mutations were detectable prior to relapse in a patient (IVL5). White circles with solid black line represent PRDM1 mutation in cell-free DNAs; Red triangles with dashed line represent Y196C CD79B in cellfree DNAs; Blue X-marks with dotted line represent levels of soluble interleukin-2 receptor. VAF, Variant allele frequencies; sIL2R, soluble interleukin-2 receptor; R-CHOP, rituximab, cyclophosphamide, doxorubicin, vincristine, prednisone; CR, complete response; IVLBCL, intravascular large B-cell lymphoma; ND, Not detected. D. L199P CD79B and PRDM1 mutations were detectable prior to relapse as extranodal diffuse large B-cell lymphoma of the central nervous system (IVL6). White circles with solid black line represent PRDM1 mutation in cell-free DNAs; Red triangles with dashed line represent L199P CD79B in cell-free DNAs; Blue X-marks with dotted line represent levels of soluble interleukin-2 receptor. VAF, Variant allele frequencies; sIL2R, soluble interleukin-2 receptor; R-CHOP, rituximab, cyclophosphamide, doxorubicin, vincristine, prednisone; CR, complete response; CNS, central nervous system; ND, Not detected. 
leg-type $(60 \%)$, primary testicular DLBCL, and primary breast DLBCL. ${ }^{1}$ The CD79B mutations, especially in p.Tyr196 (Y196 CD79B), were also recurrently found in our study, although the small number of samples tested limits the accuracy of an estimation of frequencies. These mutations have been reported in non-GCB nodal and extranodal DLBCL, similar to MYD88 L265P., ${ }^{1,8,9}$ PIM1 is known as a target of aberrant somatic hypermutation (aSHM), and its mutations have been reported in $43 \%$ of DLBCL. 10

Our targeted sequencing data showed higher VAFs in cfDNAs than in tdDNAs. Correspondingly, the sensitivity of mutation detection with liquid biopsy was $100 \%$ for the samples from those whose tdDNAs showed positive results. Furthermore, $45 \%$ of variants (15/33) were detected only in cfDNAs, but not in tdDNAs, with targeted sequencing. In studies of liquid biopsy for both unselected DLBCL and PCNSL, tdDNA showed higher mean VAFs than cfDNA (mean VAFs in tdDNA vs. in cfDNA; $35 \%$ vs. $11 \%$ in DLBCL, $47 \%$ vs. $4.7 \%$ in PCNSL). ${ }^{11,12}$ Correspondingly, the sensitivity to detect tdDNA-identified mutations in cfDNA was lower than our current result $(32 \%$ in PCNSL and $85 \%$ in DLBCL vs. $100 \%$ in IVLBCL). ${ }^{11,12}$ Based on this contrast, IVLBCL may be unusual in that cfDNA is a particularly excellent source of tumor cell-derived DNA, despite the fact that VAFs in tdDNAs were generally low due to the low tumor cell burden in the diagnostic specimens.

Because of the high sensitivity of liquid biopsy and possible high frequencies of L265P MYD88 and Y196 CD79B in IVLBCL, methods detecting these hotspot mutations in cfDNA could be highly useful diagnostic tools in the clinical setting. For example, differential diagnoses between B-cell lymphoma and other diseases are of particular value. Because these hotspots are also found in other Bcell lymphomas as described, the detection of these mutations only implies that the patient is likely to have B-cell lymphoma, without necessarily suggesting IVLBCL. However, discriminating IVLBCL from other B-cell lymphomas is often made easier by clinical manifestations including lack of lymphadenopathy and tumor formation.

In all the 3 patients, we were able to detect increasing VAFs in cfDNA prior to diagnosis of relapse (Figure 2B2D), indicating the usefulness of liquid biopsy to monitor minimal residual disease (MRD) in some IVLBCL. Since mutation analysis via ddPCR seems to have higher sensitivity than that via next-generation sequencing, ${ }^{13}$ ddPCR may therefore enable early detection of MRD.

Previous studies reported significantly higher cfDNA concentrations in serum than in plasma during the clotting process in collection tubes. ${ }^{14}$ Lower VAFs in serum could be explained by the contamination of gDNAs from circulating leukocytes.

If these mutations were frequent in healthy, aged individuals, the risk of false positivity would have to be considered. However, this is unlikely because, in a large-scale study, L265P MYD88 and Y196 CD79B were found only in $1 / 12380$ and $0 / 12380$ with the cut-off value of $2 \%{ }^{15}$ Because our sequencing analysis was not comprehensive, it is possible that more suitable for mutational analysis could be found. However, we describe the prevalence of mutations in genes involved in BCR/TLR/IL-1R/NFKB signaling pathways in IVLBCL. Furthermore, our analysis suggests that tumor cell-derived DNA is abundant in serum/plasma. Therefore, liquid biopsy to detect mutations of L265P MYD88 and Y196 CD79B may provide a powerful tool for approaching diagnosis of IVLBCL.

Yasuhito Suehara, ${ }^{1}$ Mamiko Sakata-Yanagimoto, ${ }^{2,3}$
Keiichiro Hattori, ${ }^{1}$ Toru Nanmoku, ${ }^{4}$ Takayoshi Itoh,

Daisuke Kaji, ${ }^{1,6}$ Go Yamamoto, ${ }^{6}$ Yoshiaki Abe,

Kentaro Narita, ${ }^{7}$ Masami Takeuchi, ${ }^{7}$ Kosei Matsue,

Taiki Sato, ${ }^{8}$ Masayuki Noguchi, Naoko Baba, ${ }^{2}$

Tatsuhiro Sakamoto, ${ }^{2}$ Manabu Kusakabe, ${ }^{2,3}$ Naoki Kurita, ${ }^{2,3}$

Takayasu Kato, 2,3,4 Yasuhisa Yokoyama, ${ }^{2,3}$ Hidekazu

Nishikii,, Naoshi Obara, ${ }^{2,3}$ Yuichi Hasegawa and Shigeru Chiba ${ }^{2,3,9}$

${ }^{1}$ Department of Hematology, Graduate School of Comprehensive Human Sciences, University of Tsukuba, Ibaraki; ${ }^{2}$ Department of Hematology, University of Tsukuba Hospital, Ibaraki; ${ }^{3}$ Department of Hematology, Faculty of Medicine, University of Tsukuba, Ibaraki; ${ }^{4}$ Department of Clinical Laboratory, University of Tsukuba Hospital, Ibaraki; 'Department of Hematology, JA Toride Medical Center, Toride, Ibaraki; ${ }^{6}$ Department of Hematology, Toranomon Hospital, Tokyo; ${ }^{7}$ Division of Hematology, Department of Internal Medicine, Kameda Medical Center, Kamogawa, Chiba; ${ }^{8}$ Department of Pathology, Faculty of Medicine, University of Tsukuba and ${ }^{2}$ Life Science Center of Tsukuba Advanced Research Alliance,

University of Tsukuba, Ibaraki, Japan

Correspondence: schiba-tky@umin.net doi:10.3324/haematol.2017.178830

Information on authorship, contributions, and financial \& other disclosures was provided by the authors and is available with the online version of this article at wWw. haematologica.org.

\section{References}

1. Swerdlow SH, Campo E, Harris NL, et al. WHO Classification of Tumours of Haematopoietic and Lymphoid Tissues. Revised 4th ed. Lyon, France: IARC, 2017

2. Scherer F, Kurtz DM, Newman AM, et al. Distinct biological subtypes and patterns of genome evolution in lymphoma revealed by circulating tumor DNA. Sci Transl Med. 2016;8(364):364ra155.

3. Davis RE, Ngo VN, Lenz G, et al. Chronic active B-cell-receptor signalling in diffuse large B-cell lymphoma. Nature. 2010;463(7277):8892.

4. Ngo VN, Young RM, Schmitz R, et al. Oncogenically active MYD88 mutations in human lymphoma. Nature. 2011;470(7332):115-119.

5. Compagno M, Lim WK, Grunn A, et al. Mutations of multiple genes cause deregulation of NF-kappaB in diffuse large B-cell lymphoma. Nature. 2009;459(7247):717-721

6. Lenz G, Davis RE, Ngo VN, et al. Oncogenic CARD11 mutations in human diffuse large B cell lymphoma. Science. 2008;319(5870):16761679 .

7. Matsue K, Asada N, Odawara J, et al. Random skin biopsy and bone marrow biopsy for diagnosis of intravascular large B cell lymphoma. Ann Hematol. 2011;90(4):417-421.

8. Kim Y, Ju H, Kim DH, et al. CD79B and MYD88 mutations in diffuse large B-cell lymphoma. Hum Pathol. 2014;45(3):556-564.

9. Kraan W, Horlings HM, van Keimpema M, et al. High prevalence of oncogenic MYD88 and CD79B mutations in diffuse large B-cell lymphomas presenting at immune-privileged sites. Blood Cancer J. 2013;3:e139.

10. Pasqualucci L, Neumeister P, Goossens T, et al. Hypermutation of multiple proto-oncogenes in B-cell diffuse large-cell lymphomas. Nature. 2001;412(6844):341-346.

11. Bohers E, Viailly PJ, Dubois S, et al. Somatic mutations of cell-free circulating DNA detected by next-generation sequencing reflect the genetic changes in both germinal center B-cell-like and activated Bcell-like diffuse large B-cell lymphomas at the time of diagnosis. Haematologica. 2015;100(7):e280-284.

12. Fontanilles M, Marguet F, Bohers E, et al. Non-invasive detection of somatic mutations using next-generation sequencing in primary central nervous system lymphoma. Oncotarget. 2017;8(29):4815748168.

13. Camus V, Sarafan-Vasseur N, Bohers E, et al. Digital PCR for quantification of recurrent and potentially actionable somatic mutations in circulating free DNA from patients with diffuse large B-cell lymphoma. Leuk Lymphoma. 2016;57(9):2171-2179.

14. El Messaoudi S, Rolet F, Mouliere F, Thierry AR. Circulating cell free DNA: Preanalytical considerations. Clin Chim Acta. 2013;424:222 230 .

15. Genovese G, Kahler AK, Handsaker RE, et al. Clonal hematopoiesis and blood-cancer risk inferred from blood DNA sequence. N Engl J Med. 2014;371(26):2477-2487 